\title{
STRANI FENOVENI
}

\author{
OSSER VATI \\ SULL'UOVO DI GALLINA
}

II E MOR I A

DEL GAV. PROF, VINGENZO DIORIO

ESTRATTO DAGLI ATT IELL' ACCADEMLA POVTIFICLA DE NUOVI LMCEI, ANO XXIV, SESSIONE 111. DEL 7 MAGGIO 1871.

\section{R O MI A}

THPOLRALIA DELLE SCIENZE MATEMATICHE E FISICHE

Via Lata, No. 211 A.

1871 


\section{Digitized by the Internet Archive in 2016}




\section{STRANI FENOMENI \\ OSSERVATI NELL'UOVO DELLA GALLINA DONIESTICA}

$\mathbf{H}_{\mathrm{o}}$

l'onore di richiamare l'attenzione dell'Accademia su due strani fenomeni accaduti, per quanto se ne disse a me, spontaneamente nelle uova della Gallina domestica (Phasianus gallus, Foem. Lin.). Questi per verità costituirebbero, l'uno un fatto, e l'altro un problema che, a mio credere, attendera ancor Inngamente prima di essere risoluto dalla scienza. Consiste il primo in un uovo che presenta dipinta sulla superficie del guscio l'area vascolare sanguigna, la quale cinge l'area germinativa dentro delle uova ingallate, alla fine del secondo giorno d'incubazione (1). Si riduce il secondo ad un uovicino tutto completo, ció̀ a dire munito fińninco del guscio suo calcareo, clue mi si volle persuadere da persona autorevole, rinvenuto così dentro di un uovo ordinario, rotto a caso per gli usi della economia domestica, sullo scorcio dell'A prile passato. Intorno all'uno ed all'altro preteso fenomeno, dirò succintamente il mio opiuamento.

Per quanto io mi sappia, nessun fiśologo lia fissato la suá attenzione iritorno al primo fatto annunziato. Ne è già ch'io lo creda difficile ad accadere ne'nostri climi, e con le costumanze de'nostri proventieri, sopratutto in certe stagioni dell'anno; ma si lo ritengo fra quei molti clie passano inavvertiti e forse ignoti ai cultori delle scienze naturali, che avvezzi a ricercare fra più sublimi sfere i temi de’loro studj, si dispensano talora dallo esaminare la natura negli argomenti, i quali non perciò che sieno più umili riescono sempre meno interessanti. Ed a non dipartirmi dall' esempio avanzato, chi mai potrà non meravigliarsi scorgendo disegnate dalla natıra sù di un guscio calcareo le traccie sensibili di quelle operazioni organico-vitali, clre compiendosi ove lo sguardo umano non può giungere, ci rivelano agli occhi la vita operante con misterioso artifizio nell'uovo incubato, dentro del quale si và nascostamente organizzando un essere novello? I fisiologi costretti a strappare dalle vittime sacrificate alla scienza, il verdetto di una vita che

(1) Debbo alla gentilezza del collega Prof. Socrate Cadet il possesso di questa anomalia. 
fugge loro sott' occhio, non isgradiranno adumque di vedere per tal modo, confermato dalla natura stessa, uno dei fatti più interessanti della embriologia, lo sviluppo ciò del sangue e dei vasi che lo canalizzano, e quindi lo stabilimento di una circolazione primordiale nel pulcino; laddose nè vasi, nè sanguc si rinvenivano frá le membrane o negli unori eutro de’ quali il rorpicciuolo suo si manifesta: ne è a dire che in tal caso dal di fuori quel umore gli possa essere trasfuso. Lo che se non rivela la esistenza di una forza propria, passata ereditariamente al novello essere dalla materia organizzata cle per mandato del Fattore supremo ne è la posseditrice esclusiva, forza sola capace di avvisare i corpi nei quali alherga; non saprei io da che mai trarre argomenti più efficaci a costatarne la indubitabile esistenza. Ma non volendo adesso occuparmi fuori di luogo di argomenti di filosofia zoologica, rado senza altro alla considerazione del fenomeno indicato.

Il genio immortale del bolognese Harcello Malpighi nella lettera che diresse nell'ottobre dell' anno $\mathbf{1 6 7 2}$ alla Reale Società di Londra dando notizia delfe interessantissime sue osservazioni sulla formazione del pulcino nell'novo, descrivendo la cicatricetta di un uovo dopo il secondo giorno d'incubazione, si esprimeva cosi: "In medio umbilicalis area C. locabatur (fig. 24), quac limbi "D, loco zonam albam habebat, et convexa ibidem reddebatur. Hacc reticu"Jari vasorum opere E. contegebatur, in quibus rubiginosus bumor turge" bat. Carina, solitis praedita vesiculis et corde, inratabat in colliquamento. "Spatium quod inter circulos A. et umbilicalem aream C. extcudeliatur, in" signibus risulis F. alluebatur; in dextro enim latere veluti a tot distinn ctis fontibus deducebantur aircoli G. qui ampliores redditi sul) area um" lilicali ultimum sortichantur terminnm: contentus ichor rubiginosus crat 2. et cjusdem pene naturac ac in corde et vasis observabatur; unde pulcher" rinum occurrclat spectaculum " (1). Nel caso nostro si stavano evidentemente sviluppando nell' novo da oltre a due di, ggli effetti di una di quelle incubazioni spontanec delle quali paga lo sconto l'ccomomia domestica sempre e talora eziandio l'umana salute, per l'industıe opera dei procaccini che recano sul mercato nei mesi estivi, per la publica consumazione, taluni generi di cui non si conosce nè lá data, nè il luogo di provenienza, nè de condizioni in cui spesso dis una stagione all' altra si temero conservati con lo scopo di mantenerne più elevato il prezzo.

Il guscio dell'novo del quale mi vado occupando rivela, s'io mal non mi

(1) Marcelli Mafpighi, Op. ounn. Lagduni Batav. 1637. Tom. II, pag. 79. 
appongo, l' opera inoltrata della putrefazione dopo che le circostanze ebbero interrotto dentro del medesimo il già incominciato sviluppo del germe. Questo per la sospesa spontanea incubazione essendo morto sul nascere, subi le conseguenze necessarie dell' intercettamento della vita, prima di giungere a fruirne alcuno dei compersi. I gas sviluppati nell'uovo cosi alterato avendo accresciuta l'esosmosi, che a traverso delle membrane dell' uovo naturalmente avea luogo, spinse forse le correnti del fluido sanguigno novello a traverso delle trame vascolari delicatissime. Laonde trasmesso quel fluido, coIorato per la prima volta, ed imbrunito probabilmente di più dalla reazione spiegata sul medesimo dal gas idrogeno-solforato, alle membrane del guscio dalle correnti esosmotiche; si fece strada fra le porosità esistenti nello strato calcareo che costituisce il guscio dell'novo, e diè luogo cosi a quella figura ana tomica che si vede dipinta sopra il medesimo. Attrilunisco alla stessa cagione, ossia ai gas della putrefazione, l'impronta dell'area circolare che si vede tracciata sul polo maggiore dell'uovo (1). Corrisponde la medesima alla camera d'aria, nella ruale la raccolta gassosa essendo ristretta cntro limiti più angusti, fece maggior violenza contro la parte corrispondente del guscio ; e risultato di quella pressione si fù che la crosta calcarea incominciasse già a cedere, distaccandosi parzialmente dal rimanente, siccome si vede sull' esemplare che ahbiam sott'occhio e nella annessa tavola viene rappresentato.

Ritornando ora alla faccia principale del nostro uovo dipinto dalla natura (2), se ne scorge il centro occupato da un area ellittica scura, in mezzo a cui ed alquanto lateggiata, apparisce una listerella biancastra, la quale fa si che la prima prenda l' aspetto di un corpicciuolo piegato ad arco, cou una estremità rigonfia, con l'altra acuminata, e con il seno aperto. Tre cerchi eccentrici la circondano. Si staccano dal più interno di questi, a modo di raggi asimmetrici 18 tronchi vascolari, dei quali alcuni camminano come appaiati, ed altri solitarii. Fra gli uni e gli altri decorrono vasi anastomotici che li fanno comunicare scambievolmente fra loro non solo, ma eziandio con i cerchi vascolari esterni, coi quali si vanno quei canali serpeggianti intersecando. Vi si scorge un picciol tronco che mette in comunicazione il secondo dei tre cerchi vascolari suddetti con il terzo od esterno; come pure un altro ve ne ha, che da quest'ultimo si spicca a dirittura per perdersi nell'ombra insieme con gli altri. I vasi usciti dalla cinta vascolare

(1) Vedi la tavola annessa, fig. C.

(2) Vedi la tavola stessa fig. A. 
esterna, vanno irraggiando ed irretiscono cosi tutta la superficic che chiameremo anteriore dell' uovo, mentre due dei medesimi veggonsi in penomhra, superato il polo più ristretto, disperdersi nella superficie opposta. Qnesta (1) apparisce come marmorizzata per chiazze oscure non ben definite, clie quà traspariscono discrete, e là confluenti. Se noi non andiamo errati, lo sviluppo della rete vascolare richiama la figura venosa, e la prima cinta an. mulare che circonda l'area ellittica suddetta, ricorda la sena terminalis delI'Ocken.

Gli artifizi adoperati lagli istiologi onde giungere a scuoprire i passi che d'ora in ora l'incubazione va tracciando con gli organici sviluppi dentro l' novo fecondato della gallina, ed i frutti ulsertosi che si raccolsero dalle loro ricerche, appartengono già alla scienza. Il fenomeno che noi presentiamo non serve dunque che a confermare quello ch' era già conosciuto. Ma ma prova di tal genere, mentre giova ad aggiunger fede a quanto venne già discoperto da abilissimi naturalisti, rivela forse una nuova sorgente di osservazioni; che quanto più facili ad eseguirsi, altrettanto più utili forse addiverrebbero per la scienza, e probabilmento non infruttuose per le applicazioni che ne potrebbero fare le scienze economiche e salutari.

Il celebre delle Chiaje scrivea nel 1836, che lo studio dello sviluppo del pulcino è nato e cresciuto in Italia (2). Non fù però tra noi che giunse questo alla sua maturità. L'istologia clie s'innalzò in quest' ultimi tempi moderatrice prepotente di presso che tutte le scienze naturali e biotiche, non ha percorso ancora tutto il campo che gli i destinato; c forse taluni di quei fatti che riteniamo come stranissimi e fenomenali, potrà succedere che sieno meglio compresi, e forse anco scientificamente interpretati, in un prossimo avenire. Ciò non pertanto alcuni se ne annoverano cui si vorrebbe pure trovar posto nella scienza ma sembrano destinati a rimanerne perpetuamente esclusi, almeno infmo che le nozioni che abjuamo sulla vita operante negli organismi ed intorno alle funzioni degli organi che la servano, non subiscano un totale sconvolgimento ed un rinnovamento quale non può prevedersi ancora.

Un esempio di questa ultima verita crediamo noi lo si trovi nell'uoricino che presento, il quale costituisce il secondo dei fenomeni de'quali ho inteso parlare.

Un domestico del chnno sig. Avrocato G. B. S. rompendo degli novi di

(1) Vedi la tavola, fig. B.

(2) Istituzioni di anatomia comparata. Napoli 1836. Tomo II, pag. 18.4. 
gallina per uso culinario, dicesi che rinvenisse racchiuso in uno di essi un corpicciuole, che disceso con la massa liquida che l'involgevat in un sottoposto recipiente, venne riconosciuto per un uovicino tutto formato, il quale presentato al padrone di casa, fù dal medesimo rimesso gentilmente al mio studio siccome fenonteno curioso e strano. L'uovicino speditomi è quello stesso che presento. Misura 15 mill. nel maggior diametro ossia nel bipolare, 12 ne ha nel trasverso maggiore e 10 in quello che tagliando quest'ultimo ad angolo retto, ne lo spartisse in porzioni eguali. L'uovicino è fesso verso il maggior polo irregolarmente; sicchè questa estremità dell' novo apparisce con una boccuccia shadigliante circoscritta da labruccic irregolarmente frastagliate. Da questa fenditura è fácile il riconoscere che vi fù racchiuso un torlo ed uno albume, e che la membranca testacea non vi facea difetto. Il guscio calcareo è candido ed immacolato, e lo insieme dell' novicino ricorda le uova dei Canarii (Fringilla canaria L.) chiare o non ingallate, facili ad osservarsi uclle cove di questi uccellini addomesticati. Una femmina di (juesti fino dal 12 di aprile $p^{\circ} p^{\circ}$ : emise le prime nova in mia casa. Ora senza le assertive contrarie del domestico del sig. Avvocato suddetto, si direbbe che uno di questi uovicini passato per scamottaggio dentro quello di una gallina, menire si rompeva per farne frittata, fosse addivenuto il soggetto di un preteso fenomeno naturale inesplicabile. Non volendo però noi fondarci sopra supposti di tal genere, toglieremo invece a considerare la questione dal lato scientifico; e memori della storia del dente d'oro che in altra epoca $110 n$ lontana tenne lungamente sospesi gli animi degli scienziati, vedremo se sia possibile il dare del supposto fatto una scientifica spiegazione: e cosi il ricordare le scoperte della scienza intorno ad argomenti interessantissimi, ci compenserà del tempo che vi spendiam sopra, che altrimenti avrebbe da ritenersi per inutilmente sprecato.

Velle uova chiuse in altre uova, vi sono moltissimi esempi registrati nella scicnza, e non ne mancano dei più strani e più curiosi di feti rinvenuti dentro di altri feti, come si hanno pure storic di feti o germi sviluppati entro corpi adulti, in individui nei quali la ragion del sesso si opponeva a qualsiasi altra naturale interpretazione (1). Nelle uova di gallina che adoperiamo nella economia domestica non è raro di rinvenire due torli chiusi in un guscio stesso, e qualche scrittore ricorda, di averne rinvenuti fino tre insieme. In

(1) Isidore Geoffroy Saint-Hilaire. Histoire générale et particulière des anomalies de l'organisation. Paris 1836. Tome Troisième rag. 582. 
quanto a ciò adunque nulla vi arrebbe di strano nel caso nostro astrattamente considerato. Rinvenire peraltro chiuso e sepolto nello albume di un novo di gallina normale e perfetto un altr'uovo, fornito egli pure del suo allume, e quello che è più, del suo guscio calcareo indurito, è talc un fenomeno che rijugna decisamente colle leggi anatomiche e fisiologiche funora conosciute, e somigliereble quindi ad un prodigio.

Ed invero ammettendo pure che nel caso nostro fosse avvenuto come negli altri casi accenmati, il distacco simultaneo di due torli dall' ovajo della gallina; sempre rimarreble a spiegare il come ciò avesse potuto fisiologicamente avvenire, in torli fra loro distantissimi per la differenza dello sviluppo. Da poichè la notevolissima disproporzione che vi ha fra i diamecri del uovo continente e quelli del contenuto, mette in evidenza che già la caduta simultanca di cntrambi nell'ovidotto costituirebbe un primo paradosso. Infatti oggi ‘ positivamente conosciuto che il distacco delle uova dall'ovajo, avvicne uclla gallina secondo una legge prestabilita, per la quale nessmu uovo può laccrare i vincoli che lo rattengono sullo stroma ovarico, se prima nou é giunto a quel grado di pienezza che non consentendo più agli invogli esterni di contencrlo, se ne spriggiona ed è raccolto cosi dal piccolo padiglione dell'ovidotto, che automaticamente si gitta sù lui per riceverlo ed assorbirlo nella sua via. Riesce quindi troppo astruso il comprendere, come un uovicino ad un grado relativamente minimo di sviluppo, abbia mai potuto concepire tale cnergia di morimento iniziale, da precipilare la sua parabola rincendone lutte le contrarie naturali resistenze. Ricorderemo a tale proposito una scntenza dal Leydig cspressa cosi : "Comme dans les autres classes des vertébrès, les oiseaux, les amphibies et les poissons, les oenfs ¿ maturité surpassent en grosseur ceux des mumifères; l'oraire, abstraction faite des ses autres dimensions, présente tonjours un aspect acineux, et les follicules se détachent de la masse du stroma en restant suspendus par un simple pédicule " (1).

Ma scguiamo il nostro esame. Gli autori hanno scritto che l'uovo della gallina scendendo ncll'ovidotto vi camina a rilento, ed ivi riceve lo albume che lo completa, e le membranc che lo ricuoprono; sulla più csterna delle quali si aggiunge poi verso l'estremita inferiore dell' ovidollo, per sopraposizione sotto forma di laminette cristallinc csagonali, il carbonato calcarco che in-

(1) Franz Leydig. Traité d' histologie traduit de l'allemande par R. Lahillonne. Paris 1566 , prag. 570 . 
durito ne costituisce il guscio esterno. Ora il nostro uovicino come mai avrebhe potuto pervenire a cingersi dentro lo albume dell'novo continente, di propric membrane? come sulla esterna di queste, si sarebbe mai raccolto il carbonato calcareo più o men puro, senza del quale il gnscio delle ova non si costituisce? Se il contatto immediato delle pareti secernenti di questi sali solidificabili con l'uovicino clı deve rimanerne incrostato, è condizione indispensabile perchè l' novo si completi secondo l'ordine di natura; chi potrà mai immaginare il modo, nel quale questo incrostamento si sarebbe verificato, ove realmente il picciuol uovo fosse rimasto chiuso in mezzo al liquido albuminoso di quello maggiore, e quindi per mezzo di tulte le membrane proprie di questo, separato e disgiunto dalle pareti dell' ovidotto alle quali l'escrezione di quei sali calcarei, è dimostrato che esclusivamente si appartiene? Dunque o il fatto a noi riferito è falso; o tutte le teorie e dimostrazioni della scienza, fino qui ritenute per vere sono manchevoli ed erronee. Impertanto se non rogliamo ammettere la prima parte del dilemma, conviene che necessariamente ne accettiamo la seconda. Prima però di cosi fare, ci sia lecito di riassumere quello che la scienza positiva ce ne ha tranandato.

L'unico ovajo di cui va munita la femmina degli uccelli, ritrovasi sotto i reni aderente alla colonna vertebrale e ricoperto dal peritoneo, il quale serve insieme al medesimo di copertura, di legamento e di conduttore pei vasi arteriosi e per i nervi che vanno ad investirlo, e per le vene e per i limfatici che ne risortono. Sutto alla tunica peritoneale, ridotta al solo epitelio, se ne rinviene sull'ovajo una seconda costituita da una membrana propria, la quale vien chiamata dagli anatomici l'albuginea dell'ovajo. L'aspetto generale dell' organo in attività funzionale, rassomiglia ad un grappolo di uva ad acini disegualmente sviluppati, sicchè sempre se ne scorga fra tutti uno o più maggiori, mentre si stà verificando la ovulazionc. Ciò che in talune specic non addomesticate succede ad epoche fisse dell'anno, in altre si rinnova a periodi ricorrenti e più o meno ravvicinati; ed in altre finalmente, quali sono le galline, accade quasi senz'ordine apparente; dipendendo il fenomeno juì dalle influenze del clima, della stagione, e dalle condizioni nelle quali sono esse chiamate a vivere, di quello che da una legge governata semplicemente dalla natura. Accade cosi che rel pollame la fecondità può dentro certi limiti essere messa a profitto e sviluppata, più o meno per il concorso della industria umana.

Ciascheduno di quelli acini, che dicemmo costituire con il loro insieme il 
racemo orarico, si trova aduncue ricoperto dalle due membrane che rivestono esternamente lo stroma sopra del quale si sollevarono, e giunti questi a maturanza vi sono rattenuti e legati, come indicanmo con il Leydig, da un semplice peduncolo sottilissimo, quantunque ancora tenace. A quest'epoca i vasellini sanguigni che prima vedeausi percorrere ed irretire delicatissinameute gl' invogli apparenti delle sfere ovariche sono già scomparsi e, secondo alcuni autori, una striscia biancastra equatoriale spartisce quelle in due emisferi. Noi di questa striscia biancastra nelle uova che ancora non arevano al)bandonato l'ovajo non abliamo potuto riconoscere l'evidenza; siccome pure dei pretesi calicetti ciascheduno dei quali servirebbe, come da taluni si è detto, di hase e di difesa all'novo, troviamo che il nostro delle Chiaje trovò soltanto nelle femmine degli struzzi l'esempio (1).

Queste sfere, che si chianano nel linguaggio volgare uova non nate, si svolgono e crescono sotto degli involucri membranosi suddetti, in mezzo a quella massa di tessuto connetivo e di cellule fusiformi che costituisce la porzione corticale dello stroma ovarico, distinta dalla midollare per gli anzidetti corpicciuoli vescicolari che vi si annidano, per il difetto di fihre muscolari liscie e per il meno di vascolarizzazione. I.e vescichette ovariche sono conosciute daghi anatomici con il nome del Graaf che ne illustrò la storia negli animali mammiferi c nella specie umana fino dall'anno 1672 (2). Esse peraltro non costituiscono l'uovo propriamente detto, ma solamente il nicchio od incunabolo naturale dentro del quale quello si sviluppa, e dal quale sempre spontaneamente e talora periodicamente (siccome avviene nella specic umana) si spriggiona per la lacerazione della capsula membranosa che lo contiene: lacerazione che tiene il loggo della deiscenza dei frutti capsulari di certi vegetabili, la quale concorre in essi alla dispersione dei semi entro i confuni prescritti dalla natura. L'novicino chiuso nella veschichetta di Graaf degli animali mamuniferi fù scoperto dal De Baër nell'amo 152-. Esso è involuto da un piccolo ammasso di cellole, distinto dagli anatomici con il nome di cumulo proligero (cumulus proligerus) ed immerso in un liquido proprio, il quale distende tutta la vescichetta, limitato dal di dentro al di fuori prima da uu iuroglio epiteliale che i chianato della membrana granulosa, joi in alcumi casi da una seconda capsula clie viene chiamata membrana amorfa o mem-

(1) Stefano Delle Chiaje. Istituzioni di Anatomia comparata. Scconda edizione. Napoli $1 \$ 36$, Tomo II, pag. 185.

(2) Vedi Regneri de Graaf. Opera omnia. Lugduni 1678. 
brana propria del follicolo dagli anatomici, e finalmente dall'invoglio di tunti il più esterno che costituisce la membrana esterna del follicolo di Gruaf. Questa ultima membrana è fibrosa, vascolarizzata e messa a contatto immediato con la sostanza dello stroma ovarico dentro del quale si sviluppa.

Ove però si raffrontino insieme lo sviluppo delle cellole ovariche dei mammiferi e quello che si verifica negli uccelli, ne appariscono a prina giunta notevolissime le differenze. Queste studiate comparativamente rilevano forse la sorgente di qualche equivoco sfuggito a quei scrittori di altra epoca, che ritennero per uova vere o propriamente dette sli acini, i quali cambiati in vesciche ripiene di un umore giallo caratteristico fanno di sè hella mostra sulle ovaja delle galline vendute su i mercati per uso della domestica economia, e che con proprietà di linguaggio anatomico, rappresentano le uova insieme con le capsule che le contengono.

E per verita la vescichetta di Graaf sviluppandosi nelle ovaja doppie degli animali mammiferi, incomincia dallo approfondirsi nello stroma in che naeque e si addentra sempre più nel medesimo, infino clıe la clasticità e la contrattilità dell' organo produttore e lo iniziato sviluppo delle altre cripte omonime vicine non lo risospingano in alto, sicchè debba finalmente trasparire sotto gl'invogli comuni delle oraja, in un punto qualsiasi della loro superficie. Negli uccelli invece la vescichetta stessa cresce e si svolge tutta exogena, lo clie facendo concorre a dare all' ovajo unico di fuesti animali quell' aspetto racemoso che di sopra abbiamo indicato; e quindi ognuno di quegli acini, ognuna di quelle vesciche che sù di tali organi fanmo sporgenza, non rappresenta altrimenti un uovo, ma il ricettacolo dentro del quale uno di quelli si annida. L'umore proprio della cripta Graaffiana, cambiando di natura, diviene I' umore vitellino e dà con il suo colore caratteristico l'aspetto giallongolo speciale a quelle cisti, destinate a portare al di fuor insieme con le nova racchiuse, un sacco di nutrimento copioso abjoastanza per sopperire allo sviluppo del germe, il quale nascosto in queste ultime senza ricorsi esterni potrà divenir pulcino, ove le circostanze ne favoriscano sviluppo dopo pregressa la fecondazione.

L'umor giallo che riempie la vescicola di Graaf nelle galline non è un unore omogeneo, ma fino dai primordii del suo apparire lascia intravedere, studiato al microscopio, alcune granulazioni gialle clie diremo vitelline. Queste crescendo danno a tutta la massa il loro colorito. Insieme coi granuli vitellini vi si riscontrano delle bollicine di diverso diametro, clie non sono poi altro 
che particelle olcose (olio di rosso d'uovo), e finalmente vi si trovano pure delle cellole sieno scimplici, siano nucleatc. Di queste ccllole però, a sviluppo completo dell'uovo, dobbiam dichiarare di non alverne fino ad ora vedute.

La deiscenza irregolare o la lacerazione delle cisti Graaffiane che segue l'epoca del caldo nelle femmine degli animali mammiferi c marca le ricorrenze lunari nella femmina umana, accadono pure, come indicammo, negli uccelli. In questi però tutta la vescichetta Gráffiaua lasciando l'organo ncl quale si produsse, scende insicme con il suo contenuto nell' ovidotto, che per organico eretismo si gitta ad abbraciarla. Invece negli animali mammiferi, lat cripta aperta per scissione spontanea all' epoca della orulazione, resta sull' organo ovarico uel quale nacque; e la successiva trasformazione di qucllo che fù già cumulo proligero in una sostanza gialla, ed il ritrarsi ed il ragrinsarsi dei bordi della vescicola lacerata, lasciano sulla superficic dell'organo produttore quella impronta caratteristica, a cui dalla sporgenza c dal colorito venne accordato il nome di corpo giallo corpus luteum dagli cmbriologisti. Nel caso degli uccelli accade sccondo noi, quello clic si verifica nelle piante fancrogame a frutti indeiscenti, nelle quali ritenuti quelli fino alla maturita aderenti per i pedicciuoli alla pianta produttricc; inariditi questi per il cessato passaggio degli umori, i frutti se ne distaccano al più piccolo urto, per portare i semi che gia maturi chindono nel seno a fertilizzare il scno della gran madre comune, oude riprodurre, se le circostanze non lo impediscono, le piante che prima dicdero loro la vita. Nel fatto degli animali mammiferi invece l'analogo del seme delle piante, ossia l'uovo propriamente detto, innalzato gì a maggiore potenza di sviluppo e preprarato quindi a stringere rapporti diretti con il seno materno, al quale più tardi si abharbichera se la fecondazionc non faccia difetto, abbandona spontancamente la cisti nella quale si produsse, ehe per deiscenza ne lo lascia uscire; c quindi lascia sull' ovajo la cripta che gli diè cuna, appunto cosi come veggiamo accadere in taluni vegetabili, a frutti deiscenti; che slanciano lungi da se, e talora con molta rigoria, i semi prodotti, rimanendo essi stessi aperti ed inariditi sulle piante delle 'puali cbbero già rappresentata la fruttificazione. Per conseguenza negli animali mammiferi incominciano fino dai primordii vitali a stabilirsi fra il generante ed il generato quei vincoli di famiglia, che rimanendo sempre di breve durata uei bruti, si prolungano nell' uomo siffattamente da costituire per naturale necessitu- 
dine la base della dipendenza e della soggezione, e quindi il fondamento primitivo della società umana.

Se non chic il confronto fatto or ora da noi del frutto dei vegetabili con le cosi dette nova degli uccelli, patisce una assai valida eccezione per ciò che la maggior parte dei naturalisti sembra insegnare, non essere già il laceramento del peduuculo, il quale collega la vescicola Graaffiana all'ovajo degli uccelli, quello che ne opera il distacco e la caduta nell'ovidotto; ma si hene una lacerazione a modo di deiscenza irregolare della capsula esterna; la 'fuale lascerebbe aderenti sull' organo produttore l'invogli peritoneale e fibroso; mentre la vescichetta di Graaf propriamente detta libera da questi accessorii velamenti, verrebbe presa dall'apertura slabrata (ma nor sfrangiata in questi animali) dell'ovidotto. Intorno a chè domando io venia di propurre liberamente un qualche dublio. Questo saprà forse a taluno di stravaganza ma nelle scienze naturali non essendo vietato di dubitare, soprattutto quando non si ha in vista altro che il verace avanzamento della scienza, non me ue terrei per menomanente offeso. Or bene volli già accennato fino dal principio, che quella cinta cosi detta equatoriale, la quale, per sentenza di parechi autori, si scorge attorno alle nova della gallina preste ad abbandonare l'ovajo, non è sempre facile di osservarla fino che le medesime stanno allo stato di uova non nate, appese cioè allo stroma produttore. Ora ciò non potrebbe nullamente avvenire, se quella cinta indicasse con precisione il posto ove la natura la destinato che inevitabilmente accada l'apertura degli involucri più superficiali dell' novo. Avvertirò in secondo lnogo, che più volte mi è avrenuto di trovare sulle ovaja della gallina dei torli, che non tenevano a quelle altro che per un sottile peduncolo, senza dimostrare intanto nessuna traccia d'imminente laccrazione sulla loro periferia. Ricorderò in terzo luogo, che sulle ovaja stesse ho ricercato indarno i hrani di quelle membrane esterne, ossia le abbandonate spoglic della capsula Graffiana caduta prossimamente nell'ovidotto; mentre non mi fù difficile di rinvenire i pedicciuoli i quali, cred'io, recentemente avevano perduto il frutto corrispondente. In quarto luogo accennerò che il preteso limite di vascolarizzazione su quella zona equatoriale gia detta, non è a carico delle membrane esterne ovariche che ricuoprono le vescichette sporgenti, ma solo a spese della membrana esterna della vescichetta di Graaf, la quale è realmente percorsa da vasi abbondevolissimi, insino che il contenuto suo non è cangiato totalmente in massa vitcllina; ma operato questo cangiamento, tutti quei vasi spariscono. Quindi siccome, per 
le cose dette, tutt'essa (la vescichetla Graaffiana) negli uccelli si distacca dall'ovajo per cadere nell'ovidotto, tutto porta ad argomentare, che i tralci vascolari che la ritenevano unita allo stroma orarico, si debbono senza grandi sforzi lacerare, e si lacerino di fatto ad ogni ovicino che se ne disprenda: altrimenti nessuno di essi si troverebbe mai impegnato nell'ovidotto. Ora se i tramiti vascolari non valgono ad impedire il distacco di quelle vescichette dall'orajo, vorremo noi ritenere che di tanto sieno capaci quelle brigliuzze membranose delicatissime che ricuoprono gli acini del grappolo ovarico, se ad esse nè vasi, nè fibre dimostrate aggiungono tenacità e resistenza? I commentatori dell' anatomia comparata del Barone Giorgio Cuvier, nelle giunte agli scritti di quel sommo, mentre fanno cenno della lacerazione degli involucri ovarici degli uccelli in corrispondenza della cinta equatoriale anzidetta, aggiungono in quanto alle membrane delle quali dovreblero rimanere pendenti i brani dall'orajo, clıe può essere peut-étre che queste sieno riassorbite (1). Ora fondare sopra di un può essere una teoria, ritenere per riassorbibili degli elementi anatomici senza indicare le vie per le quali questo riassorbimento di solidi per quantunque minutissimi si possa operare, mentre se ne dovrebbe ripetere ogni giorno per intiere stagioni nelle galline domestiche l'occasione; e ciò senza clie nessun fisiologo sia mai riuscito a verificarlo: vale per noi quanto il dritto d'immaginare e di esporre sotto la garanzia del può essere ogni altra teor'it, ancorchè discordante da quella infino a qui alsbracciata nello scolastico insegnamento. Che se questa regga meglio dell'altra al controllo dei fatti dei quali la prima non dà spiegazione soddisfacente, potremo domandare che venga l'una all' altra sostituita nelle scientifiche disquisizioni.

Notomizzando io le uova appese ancora all'ovajo delle galline, le rinvenni costantemente ricoperte dagli invogli ovarici sopraindicati, e staccandone talora con lieve sforzo quelle più mature dai pedicciuoli che le rattenevano, le rinvenui mai sempre con l'involucri stessi. Or se ritrovo l'uovo maturo dentro dell'ovidotto, non lo posso più riconoscere per quel di prima, nè per la sua forma, nè per la sua apparenza, nè finalmente per la sua anatomica composizione. Come mai in poche ore (6 all' incirca) tanti e cosi sorprendenti cangiamenti potè in quello operare la natura? quali congegni artificiosi ri

(1) V. Cuvier. Lccons d'anatomie comparée. Truisième ćdition revue, corrigée et considérablement augmentéc. Bruxelles 1840. Tome Troisième. Pag. 384. Il passo al quale toi ci riferiamo, crediamo che appartenga al Laurillard. 
concorsero? quale potente chimismo vi cooperò ? quale forza diresse in cosi breve tempo ed in spazio così angusto, tanto svariate ed ammirabili operazioni ?

Se bastassero solo circa 6 ore di permanenza dell'uovo nell'ovidotto (1) per rifornirlo di una doppia membrana nuova, ossia di quella dello albume e dell' altra testacea, per munirlo ed incingerlo di tutta la massa allbuminosa che per tanta parte lo riempie, per svolgere verso i poli del medesimo le briglie o calaze, per rivestirlo finalmente di un guscio calcareo il quale consolida la forma cle nel canale stesso gli è data, senza che intanto si rinvenga lo stampo nel quale quest' ultima si formò ; non saprei io da vero quale altro organo rinvenire in tutta quanta la struttura animale, che per l'attività o per le meravigliose sue produzioni potesse reggere al confronto dell'oridotto. Na se questo canale tanto potea negli uccelli, perchè mai la natura uella organizzazione degli animali mammiferi provide che gl'invogli o membrane dell'uovo scendessero con il medesimo dalle vescicliette Graffiane, mentre di poteri produttivi cosi singolari eble arricchito negli uccelli l'organo analogo per struttura a quello per il quale dovea pure l'ovicino dei mammiferi compiere naturalmente il suo tragitto ? Forse che non vi era in questi ancora un utero, ove quel complemento avrebbe potuto verificarsi ?

Ma andiamo ai fatti. E assioma provato nella scienza, che la Natura ha semplicizzato, sempre che è stato possibile, i mezzi che si proponeva per il conseguimento di un determinato fine. Or bene solo ammettendo che il distacco delle uova dallovajo che le produce, si faccia negli uccelli per la semplice lacerazione del pedunculo che le ritiene, più tosto che per la scissione e per lo spogliamento degli involucri ovarici clic le ricuoprono; troveremmo noi una maniera facilissima di spiegare il completamento delle uova nell' ovidotto per endosmosi, senza bisogno di ricorrere ad una portentosa produzione di imembrane accessorie dentro di questo canale: membrane che se appariscono semplici nella loro struttura, non per questo sono men complicate per i loro rapporti, o meno necessarie per il fine al quale furono coordinate.

Incominceremo pertanto dal rammentare, che i pochi autori che si sono occupati di discuoprire il luogo e la maniera con la quale si aggiungono al torlo dell' uovo della gallina le membrane accessorie anzidette e lo albume, non sembra sieno stati sempre d'accordo fra loro. Il Dugès (2) parla della

(1) Beclard. Traité élémentaire de Physiologie humaine. Cinquieme edition. Paris 1866. Pag. 1204.

(2) Antonio Dugès. Trattato di fisiologia comparata tradotto dal Dottor F. Gístinelli. Napoli 1841. Vol. III. Pag. 227-228. 
pellicola soltoposta al guscio come di una membrana formata nella parte superiore dell'oridotto, ed aggiunge che in seguito viene il bianco o albume. A seconda delle osservazioni di Jurkinje e Valentin riportate dal Bourdach (1), sarebbe solo nella parte mezzana e più stretta, ossia nell'istmo dell'ovidotto, che si formereblic la membrana testacea alla superficie dell'embriotrofio secondario: ivi cioè il liquido albnminoso segregato produrrebhe prima dei filamenti isolati, e poi uno strato di granulazioni che si riuniscono in una membrana. Ci facciamo intanto lecito di osservare, che siccome i liquidi assumono per legge fisica costantemente la forma del recipiente nel quale si raccolgono (giacchè è vieto assioma clıe quidquid recipitur per formam continentis recipitur), se il liquido albuminoso precedesse la formazione della memhrana che sopra del medesimo rinveniamo nelle ora compiute, converreble trovare riprodotta sù di queste la forma dell'ovidotto, c non già una forma propria e costante, e per quella dovrebbero le uova apparire sotto l'aspetto di altrettanti fusi, a pancia rotondata e ad estremità assottigliate e forse anco canalicolate longitudinalmente. Lo che non avenendo, siamo obbligati a supporre che le membrane dello albume precsistano o precedano la raccolta di questo liquido attorno alla sfera del giallo, più tosto che seguirla; mentre ciò sarebbe di impossibile spiegazione, per la difficoltà accennata da noi c tratta dalle leggi invariabili della idrostatica. Quanto perciò non rimarreble più facile il concepire che il liquido albuminoso segregato abbonderolmente nell'ovidotto, passando per forza di corrente cudosmotica a traverso delle delicatissime membranuccic clie sono destinate a contenerlo, si accumulasse in questa maniera dentro del sacco a lui preparato dalla natura; ed appunto perciò clıc tale, di pareti definite e di forma e proporzioni invariabili?

Io pertanto restringerei il mio ragionare cosi. O il liquido albuminoso che costituisce il così detto hianco dell' uovo della gallina si raccoglie attorno al giallo clie ne stahilisce il centro, prima clie le membrane le quali lo ricuoprono sieno formate; o sono queste ultime membrane quelle che precedono la raccolta di quel liquido. Se si ammette il primo, non si spieglierà mai, per le cose dette, la causa determinante della forma propria delle uova: se si ricorre al secondo, converra supporre che il giallo d'uovo fino dalla prima sua discesa nell'ovidotto abbia addossate quelle membrane, che appariscono $\mathrm{P}^{\mathrm{iù}}$ tardi su del medesimo, perchè solo allora rimangono se-

(1) C. F. Burdach. Traité de Plygsiologie. Traduit par A. J. L. Jourdan. Paris 1539. Tome deuxicme prig. 405. S 341. I, 1? 
parate e distese dallo albume del quale ultimamente s'imbeverarono. E se la cosa non andasse cosi, cone mai le fibrille ed i frocchetti destinati a formare quest'ultime, potreblero riuscire a riunirsi con tanto di tenacita e di consistenza da reggere alle spinte centrifughe del liquido che le distende? Oltre a ciò rimarrebbe quasi impossibile il concepire come ginngerebbero le membrane dello albume a rassodarsi in mezzo ad un liquido, che tenderebbe sempre a disgregarne per la stessa fluidità sua gli elementi.

Nè si supponga cl’io mi abhandoni qui alle larve di una immaginazione riscaldata, supponendo negli ovidotti una forza contrattile capace di reagire validamente contro dei liquidi che insieme con l'uovo vi si trovino di passaggio. Mentre tauto la struttura anatomica, 'quanto l'azione fisiologica di questi organi cospirano a sostegno della presente tesi. L'ovidotto unico negli uccelli, siccome unico in questi è l'ovajo, si estende da esso infino alla cloaca. É costituito in priucipio da uu canale assai stretto che si allarga a proporzione che si accosta alla cloaca. In principio l'cvidutto è di pareti assai sottili ma divengono in seguito assai resistenti. In esso ci si possono notare quattro tuniche, una esterna peritoneale, che tien fisso questo condotto alla colouna vertebrale, una seconda muscolare, una ter:a cellulosa sottile, ed una quarta uuccosa che ne tappezza l'interna superficie, ed in questa ci si osservano molti ripieghi, la piic parte slei quali souo longitudinali, oude questo canale si può prestare alla uecessaria dilatazione (1). La membrana muscolare è costituita da fibre liscie, e quindi sottratta all'impero della volontà dell'animale e governata invece dalla innervazione ganglionare, che la rende ligia ed oblediente alle operazioni automatiche dell'organismo. L'ovidotto è organo di mosiunento e di formazione (2). Incomincia esso a dimostrarsi tale, portandosi con la sua estremità aperta o libera o superiore, alla ricerca dell'ovajo ed applicandovisi sopra per assorbire l'uovo al momento del suo distacco. Chez... les oiseaux... c'est ... l'extrenité ouvert, ou le pavillou de l'oviducte qui va chercher l'ovaire, et qui s'y applique pour absorber l'oeuf au monent où il en sort (3). Siegue l'ovjdotto a spiegare la sua azione muscolare colla spinta graduata con la quale va guidando l'uovo verso l'uscita della cloaca. Finisce finalmente di dimostrarla con l' esito che determina dell' novo già compiuto a traverso della

(1) Uccelli. Compendio di anatomia-fisiologico comparala. Vol. VI., pag. $32 \pi$.

(2) Burdach. Op. cit. Tome I. Pag. 170. \$. 102.

(3) Burdach. Op. cit. Tom. II. Pag. 353. \$328. 2. 
apertura inferiore dell'ovidotto stesso, la quale offre una specie di picciolo sfiutere per le briglie muscolari che lo circondano. Che l'organo poi testè menzionato sia pure laboratorio di formazione, ì messo fuori di clubbio dallo sviluppo e dalla forma propria che in esso prendono le uova, dalla massa alhuminosa che vi si seprara, dalle calazze che dentro di esso si dispiegano nell'uovo, e per ultimo dal guscio calcareo che dentro del suo perimetro lo incrosta; e ciò per nulli ripetere di quelle inembrane, le quali dicrono sufficiente argomento al ragionar nostro.

Che se mi fosse lecito di azzardare una ipotesi, vorrei io proporre modestamente il supposto seguente. L'uovo della gallina, o più propriamente la cisti vitellina che lo raccliude, giunta a maturità, per il concorso delle trazioni che sopra della medesima dispiega l'estremità aperta e su di lei addossata dill' ovidotto, si distacca dal pedicciuolo cle la mantereva aderente allo stroma ovarico che la produsse. Distaccata cosi penctra nell'ovidotto e vi rinviene il fluido allouminoso, del quale accresce con lo stimolo della sua presenza la secrezionc. Questo fluido separato dalle ghiandole mucipore dell'ovidotto stesso, prima è denso vischioso, diviene poscia più fluido c meno tenace. La cisti vitellina essendo ripiena anch'essa di un unore di densita e di natura diversa da quello che si raceglie nell'ovidotto, ne avviene che si produce per la legge discoperta dal Dutrochet, fra i due liquidiseparati da membrane organiche, una potente corrente endosmotica, per la quale il fluido abuminoso penetrando sotto alle prime membrane che ricuoprono la cisti vitellina, ne le discosta dalle pareti sottostanti; e per conseguenza to allbume si raccoglie dentro di esse. Si stabilisce cosi una sfera esterna albuminosa at torno alla sfera interna vitellina, la quale occupa il centro. Crescendo però sempre più, dentro certi limiti, l'albume fluido al disotto delle suddette membrane esterne, e dovendo questo liquido vincere la sesistenza, che in corrispondenza dell' asse trasversale della sfera vitellina presenta al suo sempre crescente aumento lo strato delle fibre circolari dell'ovidotto; ne consiegue necessariamente che lo stesso liquido albuminoso, in ragione della resistenza incontrata, si porta in direzioni opposte, ossia verso l'asse che diverrà verticale o bipolare nell'uovo perfetto; ossia quando questo avà ragginnto il sno completo sviluppo. In altri termini il liquido che costituisce il hianco dell'uovo, ra ad accumularsi nella direzione del ranale lineare dell'ovidotto: e poichè questo canale è ristretto verso il suo principio, e dilatato verso la sua fine; ne verrá per conseguenza clie la sfera 
involgente dello alloume distesa davantaggio in queste direzioni, costituirà due poli l'uno dei quali più angusto, corrisponderà alla parte più ristrelta dell'ovidotto; e l'altro più grosso, richiamerà alla mente la parte più amplia dello stesso canale. Ora ove questi dati tcoretici si applichino allo studio prattico dell'uovo della gallina, ognuno si accorgerá che la proposta ipotesi non sembra andare troppo lungi dal vero. Per noi civè potrebbe supporsi che le due esterne tuniche dell' ovajo, le quali negli nccelli si distendono per alsbracciare da ogni parte l'uovicino prodotto, semplicizzale nella loro tessitura sotto l'azione della distenzione continua e crescente, non lo abhandonino allorquando esso lasciando la sua cuna discende nell'ovidotto; ma ivi stesso accompagnandolo, ardivengano, distese dallo albume, le tuniche esteme dell'uovo. L'interna limiterebbe al di dentro quel fluido; l'esterna, ricevendo sopra di se il deposito dei cristallini calcarei, rappresenterebbe la membrana testacca ossia del guscio. La distinzione delle due tuniche si vede tracciala in natura verso l'estremita grossa dell'novo, per lo sviluppo della cosi detla camera d'aria; la quale però nelle uova recenti appena è percettibile. Il primo allume penetrato attorno alla sfera vitellina essendo, come si è detto di sopra, assai più denso di quello clıe sarà fornito dall'ovidotto in appresso, si addensa attorno a quella e la circonda co'suoi strati. Ma per lo scostamento delle tuniche esterme nella direzione dei poli dell' novo, quell'albume denso si spiega in due briglie, le quali prendono l'aspetto di calazze o spire albuminose, per lo avvolgersi che fa su stessi la sfera vitellina nella direzione dell'asse bipolare suddetto. Basta rompere un uovo recente, versandone il contenuto dentro di un bicchiere di acqua fredda, per vedere la tessitura e la direzione delle calazze. Nè voglio qui omettere che il così detto calice vascolare esistente, secondo alcuni autori, al disotto di ciascun uovo e visibile sull'ovajo dopo la caduta dell'ovicino, e che al dire dei medesimi ivi lo ritenera accoglieva, fu solo per equivoco che potic ritenersi fatto dalle membrane che rivestono l'ovajo stesso; mentre nè la sierosa, ridotta al semplice strato epiteliale, nè la fibrosa sottostante cangiata in sottilissimo velamento diafano, divengono mai membrane vascolari. Questo atributo compete invece alla membrana esterna della vescichetta di Graaf, la quale facendo parte sustanziale del follicolo dello stesso nome, ne seguita lo sviluppo e ne accompagna con le altre vicende eziandio la caduta nell' ovidotto. Ora stando cosi le cose, come potrebhe conciliarsi la teoria antica, la quale volea che proprio la deiscenza della membrana vascolare determinasse la caduta delle uova, 
con quella proposta or ora da noi, e con il fatto che prova mancare assolutamente negli uccelli quel flusso che caratterizza il caldo nelle femmine degli animali mammiferi, e con esso la deiscenza per lacerazione dei follicoli Graaffiani? Se non vi avea differenza per i fatti anatomici fra ciò cle accade nei mammiferi e quello che si osserva negli uccelli, come è clıe tale differenza negli atti fisiologici appare manifestissima? Arroge clie nella ipotesi da noi proposta, si darebbe pure facile spiegazione della cicatricetta, la quale corrisponderebbe al punto d'inserzione del tralcio vascolare, clie insieme con il pedunculo rimane divelto dallo stroma ovarico al momento della caduta dell'uovo nell'ovidotto.

Ma é tempo oramai che ritorniamo all'assunto primitivo di questa memoria, raffrontando con l'uovo cle si pretese rinvenuto dentro dell'altro, le teorie finora esposte. La spiegazione da noi immaginata per rinvenire sull' ovajo l' origine delle membrane esterne della cisti vitellina, sarebbe senza dubbio la meno favorevole ad ammettere le probalilità di quel fenomeno. Ed in fatti se ogni e singolo uovo di gallina, fino dal distaccarsi che fa dall'organo produttore, porta seco tutti gli involucri che mostrerà spiegati giunto al completo suo sviluppo; come mai potrebbe immaginarsi che l'uno di essi riuscisse a penetrare a traverso di tutte le membrane di un suo vicino, per finirvi annidato nella massa liquida che in tanta parte lo costituisce? Mla se invece queste membrane si plasmano sullo albume addensáto attorno alle sfere vitelliue, riescirebbe meno difficile il supporre cle involute due di queste cisti uello stesso umore, potessero comparire più tardi, la maggiore come involgente della minore, sotto di velamenti comuni. Ma anclie in tale ipotesi, rimareblue ancora inesplicabile il modo col quale la sfera vitcllina più piccola, appropriandosi una porzione dello alloume separato nell'ovidotto, perverrebbe a circondarsi di membrane sue proprie, in mezzo al liquido spettante all' uovo maggiore. Ma supponiamo pure che un azione coagulativa speciale, irraggiando forse dalla piccola cisti vitellina, avesse jotenza di ciò fare, cosia che io non credo. Resterebbe tuttavia a risolversi l'ultima parte del problema proposto, la quale uon ne è certamente la più facile, mentre è da ritenersi come la più decisiva jer la prima questione proposta. Il carbonato calcareo ciò, come mai arrebbe potuto foggiarsi a guscio solido e resistente dentro dell'ovicino inchiuso, se fra questo e l'organo escretore di quel principio terroso non vi cbue alcun possibile contatto? Stimeremo noi forse che pure per endosmosi i sali calcarei siensi frecipitati sulla membrana estema dell'ovicino, attraversando inpunemente il liquido circumambiente dell'uovo maggiore e senza depositarsi al 
tempo stesso sulla sfera vitellina di questo, con la quale quel corpiccinolo inchiuso sarebbe stato in necessario contatto. Certamente nessun fisiologo vorrà ammetterlo facilmente. Ed anzi affine di addimostrarlo più chiaramente, mi sia lecito il trarne da un autore non sospetto un qualche altro positivo argomento che faccia acconciamente al nostro proposito.

Fù opinione del Jacopi (1), accettata poi e ripetuta da altri autori, che l'uovo percorrendo l'ovidotto si veste dell' albume, ed in cloaca riceve quella vernice calcarea, che poi indurita ne forma il guscio. Questa opinione però non fù ammessa da fisiologi posteriori; c già il Ranzani Professore a Bologna scriveva fino dal \$821. Discendendo poi l'ovo per l'ovidotto, gli si agginnge il bianco, o sia l'albume, in un colle nembrane, ond'è questo avsolto indi il guscio (2). Il Burdach più tardi illustrò questa tesi descrivendo più accuratamente l'anatomica struttura della membrana muccosa che riveste il lume interno dell'ovidotto degli uccelli, ed analizzandone le diverse funzioni. Scrisse egli della inferiore porzione di questo canale "plus loin, on trouve une cavité ovale, plus courte, bornée à ses deux extrénités par un renflement, ayant des parois épaisses, pourvue des muscles longitudinaux et annulaires (uterus), dans la quelle l'oeuf sejourne et acquiert sa coquille » (3). Il Le Maout ricordò ancora una membrana epidermoidea che si aggiungerebbe esternamente al guscio calcareo, della quale a dir vero non ritroviamo fatta da altri menzione speciale. Sono queste le sue parole "L'oeuf, par'enu vers l'exteemité de l' oviducte, y reçoit un depôt calcaire qui se solidifie promptement, et de plus une couche épidermoide qui contient la substunce colorante homogène ou mouchetée, qu'ou observe sur les oeufs de certains oiseaux "(4). Aggiunse finalmente precisione di dettagli il Béclard nei suoi elementi di fisiologia ripetendo: ". . . lorsque l'oeuf est arrivé au tiers inférieur de l'oviducte (c'est-àdire environ six heures après sa sortie de l'ovaire chez la poule), la couche albumineuse s'enveloppe d'une membrane, d'abord transparente, qui se dédouble bientôt en deux feuillets. Le feuillet adherent ì l'albumine restera d̀ l'état de membrane; le feuillet le plus externe s'incrustera de

(1) Giuseppe Iacopi. Elementi di Fisiologia e Notomia comparativa. Napoli 1810. Parte terza pag. 135 in fine.

(2) Camillo Ranzani. Elementi di zoologia. Tomo Terzo. Parle Prima pag. 38.

(3) Burdach. Op. e Trad. citata (1837). Vol. I, pag. 178, e 104 in fine.

(4) Emm. Le Naout. Histoire naturelle des oiseaux. Paris 1853, pag. XVI. 
cristaux calcaires et formera la coque "(1). L'Hollard, confermando pure ciò stesso, dichiarò di non riconoscere nell'ovidotto degli uccelli "nessuna dilatazione che potesse essere paragonata al una saccoccia di deposito, quindi le uova non vi si trattengono clue per rivestirsi delle loro membrane accessorie (adventives), delle quali la piì esterna è costantemente un guscio duro calcareo (2). Dallo insieme delle autorità qui riportate, deve dunque ragionevolmente conchiudersene, che sulla membrana esterna delluovo si precicipitano nell'ultima porzione dell'ovidotto, quei sali calcarei che servono ad incrostarla; quantunque forse il definitivo indurimento del guscio, in alcuni casi almeno, non si operi solo che nella cloaca. Da questa infatti, eccezionalmente, sortono talora delle uova molli e sprovvedute di quella pretesa costante guarentigia del guscio indurito e resistente: al quale fenonteno concorre forse qualche argomento ancora non ben compreso di chimismo vitale. E per verità sapendosi che il carbonato calcareo non è per se stesso solubile nei liquidi, ognuno è portato a facilmente sospettare, che quello escreto sotto forma liquida dai vasellini che arrichiscono la porzione ultima della membrana muccosa dell'ovidotto, non possa uscirne a quello stato insolubile che assume poscia precipitando sulla memlnana esterna dell'uovo. E senza dubbio la perdita di un atomo di acido carbonico, quella che lo ritorna allo stato solidificabile; ed ove questa perdita sia ritardata per la insufficienza delle reazioni vitali, può sospettarsi ne avvenga allora, per il più lento rapprendersi dell'inurganico concremento, che si stampino, per cosi dire, le impronte delle sinuosità e delle pieghe che fanno sporgenza nel lume interno della cloaca sull' uovo della gallina, fenomeno che già più volte fù dato a noi di usservare.

Ora dopo tanti fatti e ragioni chi vorrà sostenere che possa accettarsi per vero senza esitanze, il preteso fenomeno di un uovo rinvenuto con il suo guscio calcareo ben rassodato e perfetto, chiuso dentro di un'altruovo, che avea come il prino i suoi involucri c la sua coccia regolarmente costituita? E vero che il Leydig ha scritto ch̀e " il peût arriver aussi que dejàs, dans le follicule ovarique il se forme autour d'elles (ossia dell'inviluppo primitivo) d'autres enveloppes ou coques souvent très complexes, dont la genèse est encore peu connue "(3) Ma ciò non ostante, non esitiamo a con-

(1) Béclard. Traité élémentaire de Physiologie humaine. Cinfuiceme édition. Paris 1866, pag. 1204.

(2) IJollaril. Précis d'anatomie comparée. Bruxclles 1836, pag. 161.

(3) Leydig. Op. e luogo cit. 
fessare, che noi ci sentiamo poco disposti ad annoverare l'esempio che ce ne vente porto, fra le ammissibili eccezioni.

Ho esaurito un debito di convenienza e di rispetto, facendo intorno al preteso fenomeno la parte dello storico: soddisfeci a quello della scicnza, esponendo le riflessioni critiche che la stranezza del fatto mi ebbe suggerite, lasciandone se non altro al tempo la decisione. "Couciossincosachè (conchiuderò con il Vullisnieri), siccome è temeriti iusoffribile, negar subito le cose nuove, cosi nou è maschia prudená abbracciarle, prima di nou averle più e piì volte disominate, ed ossevvato, se in tutto, o in parte corrispondono "l vero, se vi sono stati equivoci, se honno i primi scoperto abbastanza, se finalmente sono uniformi a tutte le leggi della natura, che suole sovente mostrace aperta una cosa, e un altra tener celata, per lasciare a piii d'uno il mevito di faticare, a molti la gloria di scopcire, e a tutti il coutento di godere uua volta svelati gli arcani suoi (1).

(1) Vallisnicri. Opere fisico-mediche. In Venezia 1733. Tomo secondo, pag. 147. 


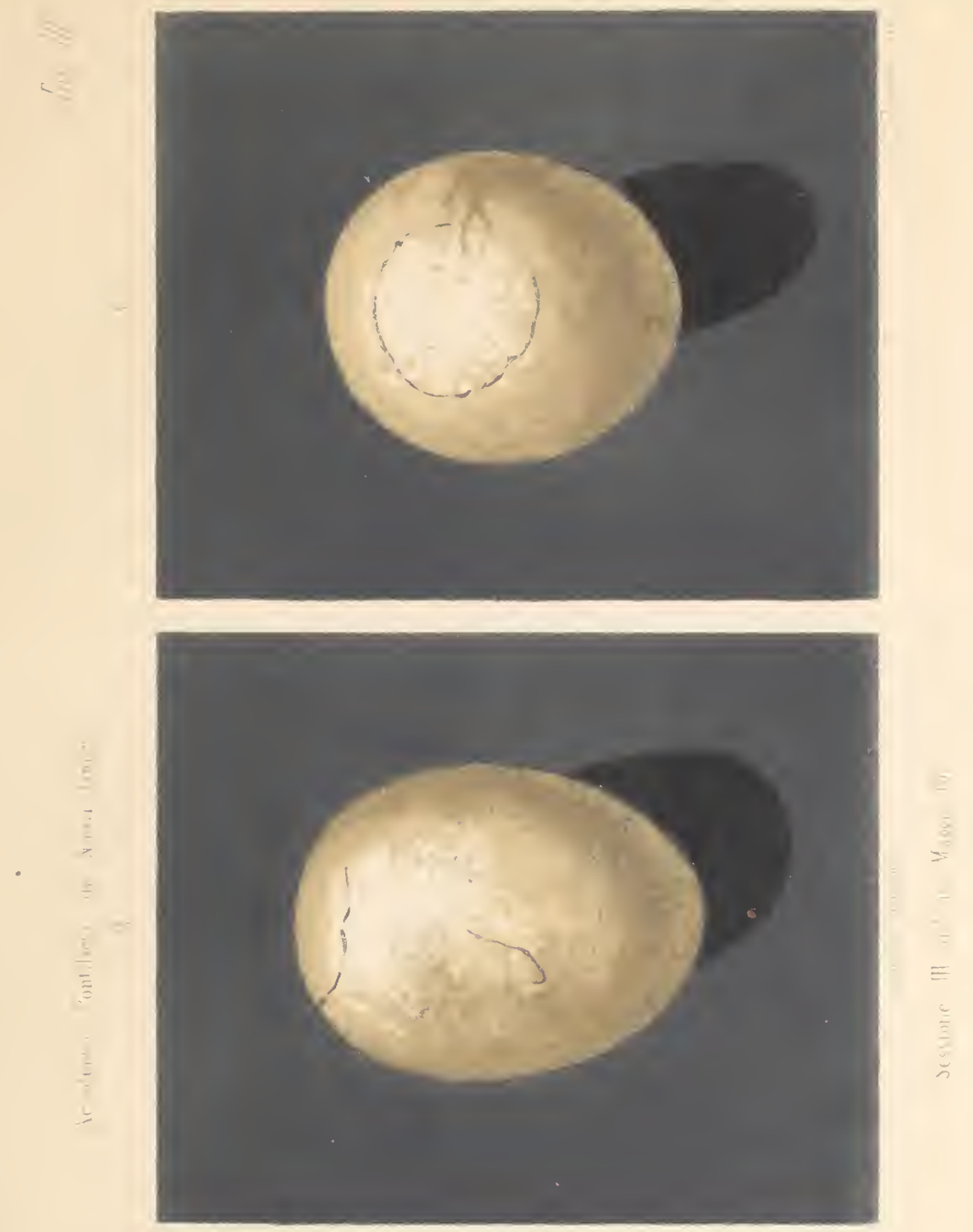
